\title{
Atomic force microscopy of membrane pore formation by cholesterol dependent cytolysins
}

Adrian W. Hodel ${ }^{a}$, Carl Leung ${ }^{a, b}$, Natalya V. Dudkina ${ }^{b}$, Helen R. Saibil ${ }^{b}$, and Bart W. Hoogenboom ${ }^{a}$

aLondon Centre for Nanotechnology, Department of Physics and Astronomy, and Institute of Structural and Molecular Biology, University College London, London WC1E 6BT, United Kingdom

${ }^{b}$ Department of Crystallography and Institute of Structural and Molecular Biology, Birkbeck College, London WC1E 7HX, United Kingdom

Corresponding author: Bart W. Hoogenboom (b.hoogenboom@ucl.ac.uk)

\section{Conflict of interest statement}

Nothing declared. 


\section{Atomic force microscopy of membrane pore formation by cholesterol dependent cytolysins}

Adrian W. Hodel $^{a}$, Carl Leung ${ }^{a, b}$, Natalya V. Dudkina $^{b}$, Helen R. Saibil ${ }^{b}$, and Bart W. Hoogenboom ${ }^{a}$ a'London Centre for Nanotechnology, Department of Physics and Astronomy, and Institute of Structural and Molecular Biology, University College London, London WC1E 6BT, United Kingdom ${ }^{b}$ Department of Crystallography and Institute of Structural and Molecular Biology, Birkbeck College, London WC1E 7HX, United Kingdom

Corresponding author: Bart W. Hoogenboom (b.hoogenboom@ucl.ac.uk)

\section{Highlights}

- Atomic force microscopy provides real-time perspective on membrane pore formation

- It has been successfully applied to cholesterol-dependent cytolysins (CDCs)

- $\mathrm{CDCs}$ are seen to transform from mobile prepore assemblies into transmembrane pores

- Ring- and arc-shaped CDC assemblies form pores in target membranes 


\title{
Atomic force microscopy of membrane pore formation by cholesterol dependent cytolysins
}

\author{
Adrian W. Hodel ${ }^{a}$, Carl Leung ${ }^{a, b}$, Natalya V. Dudkina ${ }^{b}$, Helen R. Saibil ${ }^{b}$, and Bart W. Hoogenboom ${ }^{a}$ \\ a'London Centre for Nanotechnology, Department of Physics and Astronomy, and Institute of \\ Structural and Molecular Biology, University College London, London WC1E 6BT, United Kingdom \\ ${ }^{b}$ Department of Crystallography and Institute of Structural and Molecular Biology, Birkbeck College, \\ London WC1E 7HX, United Kingdom
}

Corresponding author: Bart W. Hoogenboom (b.hoogenboom@ucl.ac.uk)

Pore forming proteins are widely used weapons of attack and defence in all domains of life. In the course of their action, they are released as monomers, bind to the target membrane, and assemble into oligomeric transmembrane pores. Our understanding of these processes has been enhanced by atomic force microscopy studies of membrane pore formation, complementing studies by other methods and by electron microscopy in particular. Here we illustrate this with recent advances in imaging pore formation by bacterial cholesterol dependent cytolysins and in elucidating their pathways of assembly on and into the membrane.

\section{Introduction}

Lipid membranes protect living cells from their surrounding environment, and inside eukaryotic cells allow for extensive compartmentalization. Damage to the cytoplasmic membrane disrupts the chemical balance of a cell, potentially leading to cell death. Many gram-positive bacteria - and at least two gram-negative bacteria [1] - exploit this to attack cell membranes with cholesterol dependent cytolysins (CDCs). These play a role in the pathogenesis of various diseases such as tissue necrosis, bacterial pneumonia, meningitis, and listeriosis [2-6].

CDCs are secreted by the bacteria as water-soluble monomers, which upon binding to the target membrane oligomerize to form transmembrane pores of 20-40 nm in diameter [7-9]. The resulting membrane disruption can be exploited by bacteria to enter host cells [10], or to enable the translocation of effectors into the target cells [11].

The structure of the water-soluble monomeric form has been solved for several CDCs by X-ray crystallography (Figure 1A, [12-19]). It appears to be well conserved through the CDC family, consists of at least 30 protein members [20], and shares the pore-forming topology with the large membrane attack complex/perforin superfamily [6]. CDC monomers comprise four domains, which are responsible for membrane binding, oligomerization, and membrane insertion. They typically bind to membranes with high $(\geq 25 \%)$ cholesterol content [21]. This binding occurs via several hydrophobic loops in domain 4 [22], after which the monomers assemble into oligomeric prepore intermediates $[2,23]$. Subsequently, the monomers undergo a dramatic structural change from the prepore into the pore state. A central beta sheet domain opens up and releases helices initially forming the transmembrane hairpin domains $\mathrm{TMH} 1$ and $\mathrm{TMH} 2$, in domain 3 . The helices unfurl and form two transmembrane beta hairpins $[7,8,24,25]$. In this process, domain 2 collapses to bring the beta strands closer to the membrane, and a large, amphipathic beta barrel is inserted into the membrane 
$[22,24,26]$. This reduces the protein height above the membrane by $4 \mathrm{~nm}[24,26,27]$. The pore transition is also accompanied by rotations of domains 1,2 , and $4[24,28]$.

Various techniques have been used to study the pathways and mechanisms of pore formation by CDCs. Negative-stain electron microscopy (EM) showed the shape and size of membrane-inserted pores $[9,24,29,30]$. Cryo-EM revealed the structures of the prepore and pore oligomeric forms, and highlighted conformational changes during prepore-to-pore transition (Figure 1B, [24,26]). Various other techniques have helped to define the mechanism of pore formation by CDCs, including fluorescence spectroscopy [2,31], fluorescence microscopy [32], site-specific mutagenesis [31], conductance measurements on black lipid membranes [33] and on live cells [34], and quartz-crystal microbalance measurements [35].

Following pioneering work on the perfringolysin $O$ [27], the past two years have seen a significant increase in the application of atomic force microscopy (AFM) to CDCs [24,35-38]. In particular when combined with detailed structural snapshots recorded by EM, AFM is a powerful tool to study the structural transitions and kinetic processes of membrane pore formation. To illustrate this, we review AFM studies of CDC pore formation and highlight how they complement results obtained by EM.

\section{AFM methods for the study of membrane pores}

Compared to other methods, a particular advantage of AFM is its ability to image label-free molecules at nanometre resolution in aqueous environment [39], allowing it to image functional biomolecules at work. AFM has been extensively used to visualise transmembrane pores, including aquaporins, gap junctions, a wide variety of ion channels, and ATP synthase [40-42], and at a larger scale the nuclear pore complex spanning the nuclear envelope of eukaryotic cells [43]. AFM has also been applied to a wide range of pore-forming toxins, as reviewed elsewhere [44].

An AFM probe comprises a sharp tip at the end of to a flexible cantilever, manufactured from siliconbased materials. In a typical AFM experiment, the tip is guided over the surface of a sample, using the deflection of the cantilever as a measure of tip-sample interactions, while tracing the surface contours. From the measured tip trace, the sample topography emerges line by line in the scanning process. This traditionally requires several minutes for the completion of an AFM image, but over the past decade the time per image - while still strongly dependent on the sample and on the required spatial resolution - has been improved to the range of seconds and below [45]. This has facilitated, for example, the study of lipid membrane domain reorganization by the pore-forming toxin lysenin [46]. Recent technological advances have also made it possible to combine high-resolution imaging with quantitative mechanical mapping [47]. Specifically, mechanical properties can be extracted from the variation of the force as a function of tip-sample distance, i.e., from so-called force-distance curves.

Most AFM and EM studies of CDCs have been carried out on model membranes. EM studies have made use of vitrified lipid vesicles for 3D structure determination [24,26], and of lipid monolayer preparations on EM grids for axial views of membrane inserted proteins [9]. Common AFM substrates such as mica facilitate the use of supported lipid bilayers [48], with two well-established methods of preparation. The Langmuir-Blodgett/Langmuir-Schaefer preparation consists of two consecutive depositions of a lipid monolayer in a Langmuir trough $[27,49,50]$. The two leaflets of the lipid bilayers can thus be of different compositions. In small Teflon wells, the resulting membrane is exposed to the protein, which binds to the membrane. The alternative and more straightforward preparation relies on lipid vesicle fusion on the AFM substrate. Under the right conditions, the lipid 
vesicles adsorb, spread out and fuse on the substrate, forming surface-covering supported lipid bilayer $[48,51,52]$, which can be exposed to pore-forming proteins.

In some cases, membrane pores have been probed or imaged on live cells [53]. Most such data have been collected on the relatively rigid peptidoglycan-rich membranes of prokaryotic cells, imaging, e.g., the porating effect of antimicrobial peptides $[54,55]$. On eukaryotic cells, it remains a challenge to image the very soft and often adhesive cytoplasmic membrane with molecular precision $[56,57]$.

\section{Visualization of membrane pore formation}

\section{Differently sized CDC assemblies perforating the membrane}

To illustrate the appearance of a transmembrane pore in AFM, Figure $1 \mathrm{C}$ shows AFM data of a single $\mathrm{CDC}$ pore, compared to the structural model derived from the cryo-EM density map (Figure 1B). The pore height ( $7 \mathrm{~nm}$ above the membrane) agrees well between the AFM surface topography and the cryo-EM structure. Features in the AFM topography appear widened compared to the structural model, because of the convolution with the finite-size AFM tip [58]. Nevertheless, sharp AFM tips can probe the pore lumen to well below the level of the surrounding membrane, thus detecting the local removal of the membrane. This can be emphasized by an appropriate choice of the false colour scale used to represent the AFM topography (Fig. 1C), and quantified via line profiles across the pore as in Figure 1D.

Using rather different sample preparations, both EM and AFM show heterogeneous distributions of CDC assemblies $[9,26,29,30,59]$, where the majority of the assemblies can be incomplete, i.e., arcinstead of ring-shaped. This is illustrated in Figure $2 A, B$ for the CDCs suilysin and listeriolysin $O$ $[24,37]$. It has long been an issue of contention whether these incomplete assemblies are functional in forming transmembrane pores [2,30]. Figure $2 A, B$ and other AFM images $[24,36,37]$ clearly demonstrate that the membrane has been removed from the lumen of arc-shaped assemblies, as well as from complete ring assemblies. In agreement with earlier evidence based on electrophysiology [33] and EM analysis [30,60], these observations imply that transmembrane pores are lined by an incomplete $\beta$-barrel (see transmembrane $\beta$-barrel in the structure in Figure $1 B, C$ ) and an unsealed lipid edge. Similar observations have been made for perforin, of the related MACPF family [61], and for Bax perforating the mitochondrial outer membrane [62].

The heterogeneity in CDC assembly size can be used to gain insight into the assembly mechanism. Distributions of oligomeric populations have been obtained from AFM and negative-stain EM images on suilysin, and found to be reproducible between the two methods (Figure 2C,D) with their respective sample preparations. These distributions can be interpreted by means of a simple model that assumes irreversible CDC binding to the membrane followed by irreversible assembly on the membrane by association of CDC monomers (or short oligomers) to grow larger assemblies [24]. In line with earlier suggestions [59], depletion of monomers on the membrane arrests the assembly process: Depending on the relative rates for membrane binding and monomer association, this can result in oligomeric populations similar to the ones observed experimentally (Figure 2C,D, grey, dashed lines). Hence the experimental data are consistent with a kinetically trapped assembly process via association of CDC monomers (or up to tetra- or pentamers, [24]) to growing assemblies. Arc-shaped pore assemblies can also be found to merge or interlock (see Figure $2 \mathrm{~A}, \mathrm{~B}(\mathrm{iii})$ ) and thus form membrane perforations of varying shape and size [24,36,37]. 
AFM greatly complements the more detailed static structural information from cryo-EM by continuously imaging the same area on the membrane while recording changes as a function of time or triggered by external factors $[24,27,38]$. The early stages of CDC assembly have thus far eluded direct visualization, but the prepore-to-pore transition has been imaged by AFM for perfringolysin 0 (Figure 3, [27]) and suilysin (Figure 4, [24]). These experiments made use of genetically engineered constructs forming a disulphide bond to trap the CDCs in the (otherwise transient) prepore state [23]. Following exposure to a reducing agent such as DL-dithiothreitol (DTT), these constructs insert into the membrane and form pores as the wild-type protein, allowing real time observation of the prepore-to-pore transition.

CDC prepores are loosely attached to the membrane via several hydrophobic loops of domain 4 [22], and can diffuse freely on the membrane. This has been confirmed by real-time AFM imaging at 15 seconds per frame with the temperature reduced to $15^{\circ} \mathrm{C}$, in which case the mobile assemblies were sufficiently slowed down to become visible by AFM (Video 1 ). If the protein concentration is significantly increased, the prepores become more densely packed on the membrane. This dense packing reduces the mobility of the prepores and allows them to be resolved by AFM as static assemblies that protrude $\approx 11 \mathrm{~nm}$ above the membrane (Figure $3 \mathrm{~A}-\mathrm{C},[24,27]$ ).

Following exposure to DTT, the CDC prepore assemblies collapse to a height of $\approx 7 \mathrm{~nm}$ above the membrane (Figure 3A-C, [27]), consistent with cryo-EM observations on wild-type pneumolysin at lower temperature [26] and on disulphide locked suilysin [24]. This reduction in height is indicative of the collapse of the hinge domain (green in Figure 1A) that coincides with the unfurling of the transmembrane hairpin domains (yellow in Figure $1 \mathrm{~A}$ ) to form the transmembrane $\beta$-barrel (yellow in Figure 1B).

These earlier data on densely packed, static assemblies [27] have more recently been complemented by sequences of AFM images that show mobile CDC prepores transiting into the pore state [24]. Visualized at lower time resolution than the data in Video 1 and at room temperature, added (disulphide-locked) protein initially appeared as diffuse streaks on the membrane (Video 2), as the protein here moves too fast to be resolved by the AFM scanning process. Nevertheless, such image sequences allow tracking of CDC pore formation step by step. Figure 4 displays cropped regions of consecutive AFM images (Video 2), showing the initial appearance of disulphide-locked CDC prepores as streaky features in the images, with an apparent height of approximately $10 \mathrm{~nm}$ above the membrane surface, indicating that there is no collapse of domain 2 ( $0 \mathrm{~min})$. Following exposure to DTT (4 min), (clusters of) prepore assemblies become immobilised, possibly indicative of yet unknown prepore intermediates or of only partially inserted pores, and next show the vertical collapse (8 $\mathrm{min}$ ), similar to perfringolysin $\mathrm{O}$ (Figure 3A-C, [27]). The vertical collapse is followed by the emergence of high features on the membrane ( $15 \mathrm{~min})$, interpreted as lipid being ejected from the membrane, and the final membrane perforation (20 $\mathrm{min})$.

To gain further insight into the nature of the prepore-to-pore transition, such data can be complemented by AFM experiments in which membrane pore formation is deliberately hampered or induced. For example, pore formation by wild-type suilysin was impeded by co-incubation with the disulphide-locked variant in a dose-dependent manner [24], whereas full functionality and pore formation were restored upon subsequent reduction of the disulphide bond. This implies that wildtype and disulphide-locked protein bind and co-assemble on the membrane, and that the presence of the locked subunits in the assembly prevents membrane pore formation. Hence membrane insertion depends on coordinated conformational changes by all (or most) subunits in an assembly. On the other hand, a controlled vertical pressure by the AFM can be applied to induce the vertical collapse of the hinge domain in prepore-locked perfringolysin $O$ assemblies [38], and to subsequently relate this to the mechanical energy that is released on the vertical collapse of the prepore. 


\section{Conclusions and outlook}

AFM has provided a new perspective on CDC pore formation by imaging the functionality of both complete and incomplete CDC assemblies in perforating lipid membranes, thus confirming the old but disputed proposition of arc-shaped pores. Its main attractiveness, however, resides in its potential to visualise the process of membrane pore formation in real time, to study and dissect pathways of pore assembly. This potential is only starting to be exploited [24,27,37], with continuously improving AFM technology and improving control, e.g., via mutagenesis or temperature, of the kinetics of pore formation. AFM can thus identify intermediate states of membrane pore formation, which can then be examined by cryo-EM for structure determination. In terms of resolution, AFM resides between EM (static views, high spatial resolution) and singlemolecule fluorescence microscopy (high temporal, low spatial resolution). Ultimately, in-depth understanding of membrane pore formation is therefore most likely to arise from a combination of these complementary methods. Such a combination is also particularly attractive because it facilitates the study of model systems (e.g., reconstituted lipid membranes) as well as of whole organelles or cells [62].

\section{Conflict of interest statement}

The authors declare no conflict of interest.

\section{Acknowledgement}

The research described here from the authors' laboratories was supported by grants from the UK Biotechnology and Biological Sciences Research Council (BB/G011729/1, BB/J005932/1, BB/J006254 and BB/K01692X/1) and from the European Research Council (294408). The authors acknowledge Estefania Mulvihill and Daniel Müller for providing the AFM data displayed in Figure 2B.

\section{References}

1. Hotze EM, Le HM, Sieber JR, Bruxvoort C, Mclnerney MJ, Tweten RK: Identification and Characterization of the First Cholesterol-Dependent Cytolysins from Gram-Negative Bacteria. Infect. Immun. 2013, 81:216-225.

2. Hotze EM, Tweten RK: Membrane assembly of the cholesterol-dependent cytolysin pore complex. Biochim. Biophys. Acta 2012, 1818:1028-1038.

3. Bischofberger M, lacovache I, van der Goot FG: Pathogenic Pore-Forming Proteins: Function and Host Response. Cell Host Microbe 2012, 12:266-275.

4. Gilbert RJC, Mikelj M, Dalla Serra M, Froelich CJ, Anderluh G: Effects of MACPF/CDC proteins on lipid membranes. Cell. Mol. Life Sci. 2013, 70:2083-2098.

5. Cassidy S, O'Riordan M: More Than a Pore: The Cellular Response to Cholesterol-Dependent Cytolysins. Toxins 2013, 5:618-636.

6. Reboul CF, Whisstock JC, Dunstone MA: Giant MACPF/CDC pore forming toxins: A class of their own. Biochim. Biophys. Acta 2015, doi:10.1016/j.bbamem.2015.11.017. 
7. Shepard LA, Heuck AP, Hamman BD, Rossjohn J, Parker MW, Ryan KR, Johnson AE, Tweten RK: Identification of a Membrane-Spanning Domain of the Thiol-Activated Pore-Forming Toxin Clostridium perfringens Perfringolysin O: An $\alpha$-Helical to $\beta$-Sheet Transition Identified by Fluorescence Spectroscopy. Biochemistry 1998, 37:14563-14574.

8. Shatursky O, Heuck AP, Shepard LA, Rossjohn J, Parker MW, Johnson AE, Tweten RK: The Mechanism of Membrane Insertion for a Cholesterol-Dependent Cytolysin: A Novel Paradigm for Pore-Forming Toxins. Cell 1999, 99:293-299.

9. Dang TX, Hotze EM, Rouiller I, Tweten RK, Wilson-Kubalek EM: Prepore to pore transition of a cholesterol-dependent cytolysin visualized by electron microscopy. J. Struct. Biol. 2005, 150:100-108.

10. Alberti-Segui C, Goeden KR, Higgins DE: Differential function of Listeria monocytogenes listeriolysin $\mathbf{O}$ and phospholipases $\mathbf{C}$ in vacuolar dissolution following cell-to-cell spread. Cell. Microbiol. 2007, 9:179-195.

11. Madden JC, Ruiz N, Caparon M: Cytolysin-Mediated Translocation (CMT): A Functional Equivalent of Type III Secretion in Gram-Positive Bacteria. Cell 2001, 104:143-152.

12. Rossjohn J, Feil SC, McKinstry WJ, Tweten RK, Parker MW: Structure of a Cholesterol-Binding, Thiol-Activated Cytolysin and a Model of Its Membrane Form. Cell 1997, 89:685-692.

13. Polekhina G, Giddings KS, Tweten RK, Parker MW: Insights into the action of the superfamily of cholesterol-dependent cytolysins from studies of intermedilysin. Proc. Natl. Acad. Sci. U. S. A. 2005, 102:600-605.

14. Bourdeau RW, Malito E, Chenal A, Bishop BL, Musch MW, Villereal ML, Chang EB, Mosser EM, Rest RF, Tang W-J: Cellular Functions and X-ray Structure of Anthrolysin O, a Cholesteroldependent Cytolysin Secreted by Bacillus anthracis. J. Biol. Chem. 2009, 284:14645-14656.

15. Xu L, Huang B, Du H, Zhang XC, Xu J, Li X, Rao Z: Crystal structure of cytotoxin protein suilysin from Streptococcus suis. Protein Cell 2010, 1:96-105.

16. Feil SC, Ascher DB, Kuiper MJ, Tweten RK, Parker MW: Structural Studies of Streptococcus pyogenes Streptolysin O Provide Insights into the Early Steps of Membrane Penetration. J. Mol. Biol. 2014, 426:785-792.

17. Köster S, van Pee K, Hudel M, Leustik M, Rhinow D, Kühlbrandt W, Chakraborty T, Yildiz Ö: Crystal structure of listeriolysin $\mathrm{O}$ reveals molecular details of oligomerization and pore formation. Nat. Commun. 2014, 5:3690.

18. Marshall JE, Faraj BHA, Gingras AR, Lonnen R, Sheikh MA, El-Mezgueldi M, Moody PCE, Andrew PW, Wallis R: The Crystal Structure of Pneumolysin at 2.0 $\AA$ Resolution Reveals the Molecular Packing of the Pre-pore Complex. Sci. Rep. 2015, 5:13293.

19. Lawrence SL, Feil SC, Morton CJ, Farrand AJ, Mulhern TD, Gorman MA, Wade KR, Tweten RK, Parker MW: Crystal structure of Streptococcus pneumoniae pneumolysin provides key insights into early steps of pore formation. Sci. Rep. 2015, 5:14352.

20. Johnson BB, Heuck AP: Perfringolysin O Structure and Mechanism of Pore Formation as a Paradigm for Cholesterol-Dependent Cytolysins. Subcell. Biochem. 2014, 80:63-81.

21. Johnson BB, Moe PC, Wang D, Rossi K, Trigatti BL, Heuck AP: Modifications in Perfringolysin 0 Domain 4 Alter the Cholesterol Concentration Threshold Required for Binding. Biochemistry 2012, 51:3373-3382.

22. Ramachandran R, Heuck AP, Tweten RK, Johnson AE: Structural insights into the membraneanchoring mechanism of a cholesterol-dependent cytolysin. Nat. Struct. Biol. 2002, 9:823- 
827.

23. Hotze EM, Wilson-Kubalek EM, Rossjohn J, Parker MW, Johnson AE, Tweten RK: Arresting Pore Formation of a Cholesterol-dependent Cytolysin by Disulfide Trapping Synchronizes the Insertion of the Transmembrane $\beta$-Sheet from a Prepore Intermediate. J. Biol. Chem. 2001, 276:8261-8268.

24. Leung C, Dudkina N V, Lukoyanova N, Hodel AW, Farabella I, Pandurangan AP, Jahan N, Pires Damaso M, Osmanović D, Reboul CF, et al.: Stepwise visualization of membrane pore formation by suilysin, a bacterial cholesterol-dependent cytolysin. Elife 2014, 3:e04247.

** This paper includes the latest structural models for CDC pores and prepores, uses a combined EM and AFM approach to visualise various steps in the process of CDC pore formation, and provides a model for CDC assembly.

25. Lukoyanova N, Kondos SC, Farabella I, Law RHP, Reboul CF, Caradoc-Davies TT, Spicer BA, Kleifeld O, Traore DAK, Ekkel SM, et al.: Conformational Changes during Pore Formation by the Perforin-Related Protein Pleurotolysin. PLOS Biol. 2015, 13:e1002049.

* Cryo EM and structural analysis of a MACPF protein in prepore and pore states traces the opening of the central beta-sheet that releases helical domains to form the transmembrane beta barrel. Movement of a key conserved motif is proposed to unlock the bent conformation.

26. Tilley SJ, Orlova E V, Gilbert RJC, Andrew PW, Saibil HR: Structural Basis of Pore Formation by the Bacterial Toxin Pneumolysin. Cell 2005, 121:247-256.

27. Czajkowsky DM, Hotze EM, Shao Z, Tweten RK: Vertical collapse of a cytolysin prepore moves its transmembrane $\beta$-hairpins to the membrane. EMBO J. 2004, 23:3206-3215.

28. Reboul CF, Whisstock JC, Dunstone MA: A New Model for Pore Formation by CholesterolDependent Cytolysins. PLOS Comput. Biol. 2014, 10:e1003791.

29. Bhakdi S, Tranum-Jensen J, Sziegoleit A: Mechanism of Membrane Damage by StreptolysinO. Infect. Immun. 1985, 47:52-60.

30. Palmer M, Harris R, Freytag C, Kehoe M, Tranum-Jensen J, Bhakdi S: Assembly mechanism of the oligomeric streptolysin $O$ pore: the early membrane lesion is lined by a free edge of the lipid membrane and is extended gradually during oligomerization. EMBO J. 1998, 17:15981605.

31. Ramachandran R, Tweten RK, Johnson AE: Membrane-dependent conformational changes initiate cholesterol-dependent cytolysin oligomerization and intersubunit $\beta$-strand alignment. Nat. Struct. Mol. Biol. 2004, 11:697-705.

32. Rampersaud R, Planet PJ, Randis TM, Kulkarni R, Aguilar JL, Lehrer RI, Ratner AJ: Inerolysin, a Cholesterol-Dependent Cytolysin Produced by Lactobacillus iners. J. Bacteriol. 2011, 193:1034-41.

33. Marchioretto $M$, Podobnik M, Dalla Serra M, Anderluh G: What planar lipid membranes tell us about the pore-forming activity of cholesterol-dependent cytolysins. Biophys. Chem. 2013, 182:64-70.

34. El-Rachkidy RG, Davies NW, Andrew PW: Pneumolysin generates multiple conductance pores in the membrane of nucleated cells. Biochem. Biophys. Res. Commun. 2008, 368:786-792.

35. Stewart SE, D’Angelo ME, Piantavigna S, Tabor RF, Martin LL, Bird PI: Assembly of streptolysin $O$ pores assessed by quartz crystal microbalance and atomic force microscopy provides evidence for the formation of anchored but incomplete oligomers. Biochim. Biophys. Acta 2015, 1848:115-126. 
36. Podobnik M, Marchioretto M, Zanetti M, Bavdek A, Kisovec M, Cajnko MM, Lunelli L, Dalla Serra M, Anderluh G: Plasticity of Lysteriolysin O Pores and its Regulation by $\mathrm{pH}$ and Unique Histidine. Sci. Rep. 2015, 5:9623.

37. Mulvihill E, van Pee K, Mari SA, Müller DJ, Yildiz Ö: Directly Observing the Lipid-Dependent Self-Assembly and Pore-Forming Mechanism of the Cytolytic Toxin Listeriolysin O. Nano Lett. 2015, 15:6965-6973.

* Mostly based on AFM data, this paper shows how CDC assembly and pore forming mechanism can depend on cholesterol and the phospholipid composition of the target membranes.

38. Czajkowsky DM, Sun J, Shen Y, Shao Z: Single molecule compression reveals intra-protein forces drive cytotoxin pore formation. Elife 2015, 4:e08421.

39. Müller DJ, Engel A: Atomic force microscopy and spectroscopy of native membrane proteins. Nat. Protoc. 2007, 2:2191-2197.

40. Müller DJ, Engel A: Strategies to prepare and characterize native membrane proteins and protein membranes by AFM. Curr. Opin. Colloid Interface Sci. 2008, 13:338-350.

41. Scheuring S, Dufrêne YF: Atomic force microscopy: probing the spatial organization, interactions and elasticity of microbial cell envelopes at molecular resolution. Mol. Microbiol. 2010, 75:1327-1336.

42. Fotiadis D: Atomic force microscopy for the study of membrane proteins. Curr. Opin. Biotechnol. 2012, 23:510-515.

43. Bestembayeva A, Kramer A, Labokha AA, Osmanović D, Liashkovich I, Orlova E V., Ford IJ, Charras G, Fassati A, Hoogenboom BW: Nanoscale stiffness topography reveals structure and mechanics of the transport barrier in intact nuclear pore complexes. Nat. Nanotechnol. 2015, 10:60-64.

44. Yilmaz N, Kobayashi T: Assemblies of pore-forming toxins visualized by atomic force microscopy. Biochim. Biophys. Acta 2015, doi:10.1016/j.bbamem.2015.11.005.

* This review provides a broad overview of the application of AFM to pore forming toxins, to understand their assembly and their effect on membrane organization.

45. Ando T, Uchihashi T, Kodera N, Yamamoto D, Miyagi A, Taniguchi M, Yamashita H: High-speed AFM and nano-visualization of biomolecular processes. Pflügers Arch. - Eur. J. Physiol. 2008, 456:211-225.

46. Yilmaz N, Kobayashi T: Visualization of Lipid Membrane Reorganization Induced by a PoreForming Toxin Using High-Speed Atomic Force Microscopy. ACS Nano 2015, 9:7960-7967.

47. Dufrêne YF, Martínez-Martín D, Medalsy I, Alsteens D, Müller DJ: Multiparametric imaging of biological systems by force-distance curve-based AFM. Nat. Methods 2013, 10:847-854.

48. Mingeot-Leclercq M-P, Deleu M, Brasseur R, Dufrêne YF: Atomic force microscopy of supported lipid bilayers. Nat. Protoc. 2008, 3:1654-1659.

49. Czajkowsky DM, Iwamoto H, Cover TL, Shao Z: The vacuolating toxin from Helicobacter pylori forms hexameric pores in lipid bilayers at low pH. Proc. Natl. Acad. Sci. U. S. A. 1999, 96:2001-2006.

50. Luo M, Shao Z, Shen Y, Czajkowsky DM, Sun J: Novel experimental strategy for high resolution AFM imaging of membrane-associated bacterial toxins. J. Shanghai Jiaotong Univ. 2014, 19:569-573. 
51. Richter R, Mukhopadhyay A, Brisson A: Pathways of Lipid Vesicle Deposition on Solid Surfaces: A Combined QCM-D and AFM Study. Biophys. J. 2003, 85:3035-3047.

52. Richter RP, Brisson AR: Following the Formation of Supported Lipid Bilayers on Mica: A Study Combining AFM, QCM-D, and Ellipsometry. Biophys. J. 2005, 88:3422-3433.

53. Müller DJ, Helenius J, Alsteens D, Dufrêne YF: Force probing surfaces of living cells to molecular resolution. Nat. Chem. Biol. 2009, 5:383-390.

54. Meincken M, Holroyd DL, Rautenbach M: Atomic Force Microscopy Study of the Effect of Antimicrobial Peptides on the Cell Envelope of Escherichia coli. Antimicrob. Agents Chemother. 2005, 49:4085-4092.

55. Fantner GE, Barbero RJ, Gray DS, Belcher AM: Kinetics of antimicrobial peptide activity measured on individual bacterial cells using high-speed atomic force microscopy. Nat. Nanotechnol. 2010, 5:280-285.

56. Eghiaian F, Rigato A, Scheuring S: Structural, Mechanical, and Dynamical Variability of the Actin Cortex in Living Cells. Biophys. J. 2015, 108:1330-1340.

57. Kronlage C, Schäfer-Herte $M$, Böning D, Oberleithner H, Fels J: Feeling for Filaments: Quantification of the Cortical Actin Web in Live Vascular Endothelium. Biophys. J. 2015, 109:687-698.

58. Pyne A, Thompson R, Leung C, Roy D, Hoogenboom BW: Single-Molecule Reconstruction of Oligonucleotide Secondary Structure by Atomic Force Microscopy. Small 2014, 10:32573261.

* By visualising and analysing the DNA double helix by AFM, this paper highlights the resolving power of AFM, as well as some of its limitations.

59. Gilbert RJC: Inactivation and Activity of Cholesterol-Dependent Cytolysins: What Structural Studies Tell Us. Structure 2005, 13:1097-1106.

60. Sonnen AF, Plitzko M, Gilbert RJC: Incomplete pneumolysin oligomers form membrane pores. 2014, [no volume].

61. Metkar SS, Marchioretto M, Antonini V, Lunelli L, Wang B, Gilbert RJC, Anderluh G, Roth R, Pooga M, Pardo J, et al.: Perforin oligomers form arcs in cellular membranes: a locus for intracellular delivery of granzymes. [Internet]. Cell Death Differ. 2015, 22:74-85.

62. Salvador-Gallego R, Mund M, Cosentino K, Schneider J, Unsay J, Schraermeyer U, Engelhardt J, Ries J, Garcia-Saez AJ: Bax assembly into rings and arcs in apoptotic mitochondria is linked to membrane pores. EMBO J. 2016, doi:10.15252/embj.201593384.

** This paper uses a combination of AFM, EM and single-molecule fluorescence microscopy to demonstate the presence of arc-shaped Bax pores in outer michochondrial membranes, thus demonstrating that such pores also exist beyond the CDC/MACPF superfamily. 


\section{Figure legends}

Figure 1. Structural studies of suilysin from Streptococcus suis. (A) Crystal structure of suilysin with the domains labelled ([15]; PDB:3hvn). (B) A cut through the cryo-EM map of the pore oligomeric form with fitted atomic structures [24]. (C) Surface topography cross-section of the pore as measured by AFM in aqueous solution, shown on a false-colour scale and in 3D representation, overlaid with the structural model from (B). (D) Height profile across the pore in (C), illustrating the protrusion of the pore from the membrane (defined as zero height) and the removal of the membrane in the pore lumen.

Figure 2. Membrane perforation by CDCs. Depending on the shape of the AFM tip and the width of the pore lumen, AFM can probe the presence/absence of the membrane inside CDC assemblies, here illustrated at the same scale for suilysin of Streptococcus suis (A) and listeriolysin $\mathrm{O}$ of Listeria monocytogenes (B). Membrane holes are formed by complete ring-shaped CDC assemblies (i), by incomplete arc-shaped CDC assemblies (ii), and by interlocked or merged CDC arc assemblies (iii). As in Figure 1, the false-colour scale $(25 \mathrm{~nm})$ is chosen such that green represents the membrane height, purple and white represent protrusions from it, and black represents indentations into the membrane. (C) and (D) show the distribution of oligomeric populations for suilysin pore assemblies, as determined from AFM on supported lipid bilayers and from negative-stain EM on lipid monolayer preparations. Grey, dashed lines indicate fits with a simple model for kinetically trapped oligomerization. The listeriolysin O data in (B) is adapted from [37] with permission. Copyright (2015) American Chemical Society. (C) and (D) are reproduced from [24].

Figure 3. Transition from prepore to pore assemblies, for $C D C$ perfringolysin $\mathrm{O}$ of Clostridium perfringens. $(A, B, C)$ Sequential AFM images of the same area with dense clusters of CDC assemblies, showing their vertical collapse from the $\sim 11 \mathrm{~nm}$ high prepore state to the $\sim 7 \mathrm{~nm}$ high pore state (heights measured with respect to the membrane surface). (D, E) Higher-magnification AFM images, resolving the $C D C$ subunits in prepore $(D)$ and pore $(E)$ assemblies. Reproduced with permission from [27]. Copyright (2004) European Molecular Biology Organization.

Figure 4. AFM tracks the sequence of events from mobile CDC prepores to membrane-perforating pores, here shown for suilysin of Streptococcus suis. Top row: Extracts from a sequence of AFM images (Video 2), with streaky features characteristic of mobile assemblies (0 min). Subsequent images show a cluster of assemblies that become immobilized ( $4 \mathrm{~min}$ ) and collapse into the membrane ( $8 \mathrm{~min}$ ). As here illustrated for another assembly in the same experiment, this is followed by the appearance of high features ( $15 \mathrm{~min}$ ) - interpreted as lipids being ejected from the membrane - and membrane perforation ( $20 \mathrm{~min}$ ) as detectable in the pore lumen. Bottom row: Corresponding line profiles (see arrows in top row images) compared to the heights of the prepore and pore structures above the membrane. The prepore-to-pore transition is triggered at $2 \mathrm{~min}$ (see text), and the red asterisk refers to the hole in the membrane. Adapted from [24]. 


\section{Legends for video files}

Video 1. Mobile CDC (suilysin) prepore assemblies diffusing on the membrane. At a temperature of $15^{\circ} \mathrm{C}$, the mobility of the prepores is sufficiently reduced for the assemblies to be resolved by realtime AFM at $15 \mathrm{~s} /$ frame. This sequence of images was captured $\sim 30$ min after protein injection and at 384 pixels per line. The timing of the video is accelerated by a factor of $\sim 100$. Full z-color scale $=20$ $\mathrm{nm}$. Reproduced from [24].

Video 2. Real-time imaging of the prepore-to-pore transition and membrane perforation by suilysin. Subsequent AFM frames of the same area were alternatively recorded from top to bottom and from bottom to top, as indicated by white arrows. Frame time: $4 \mathrm{~min}$; color scale: $35 \mathrm{~nm}$. Adapted from [24]. The prepore-to-pore transition is triggered after $2 \mathrm{~min}$. 
A

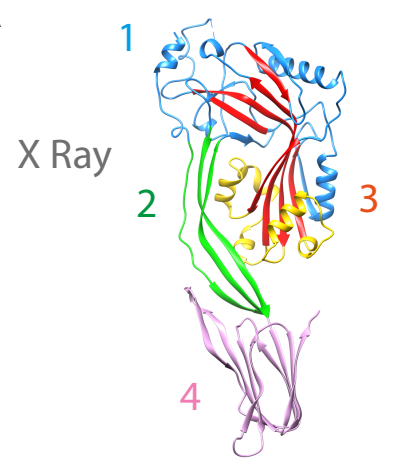

D

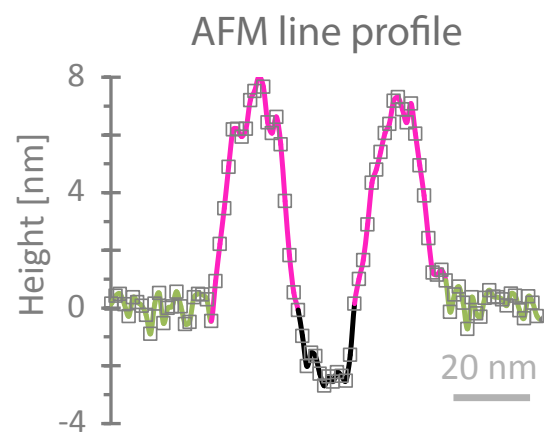

B

Cryo EM

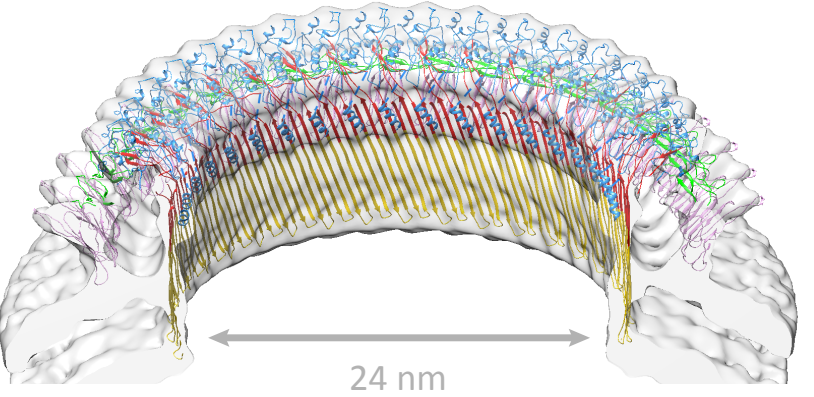

C

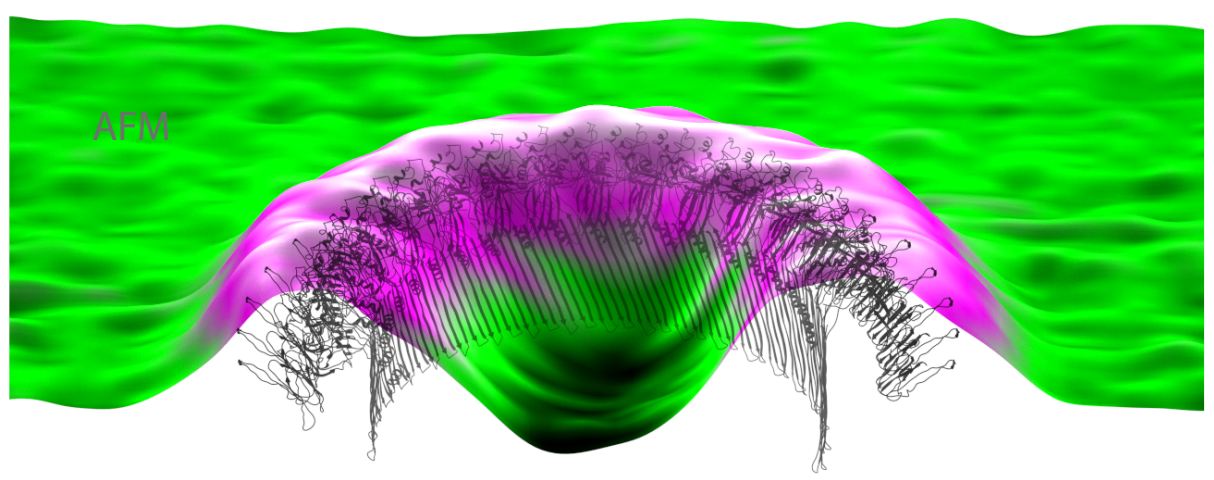




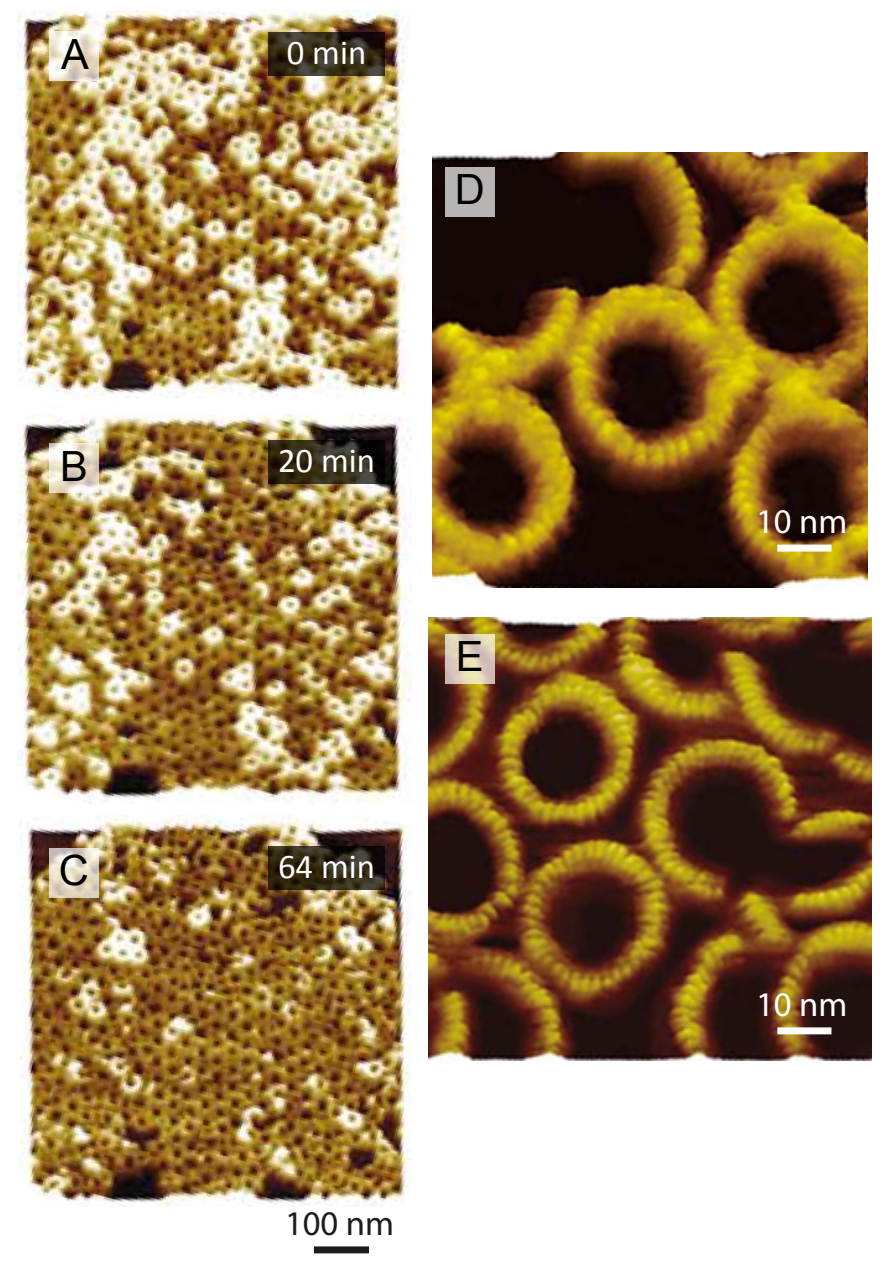

Figure 3 


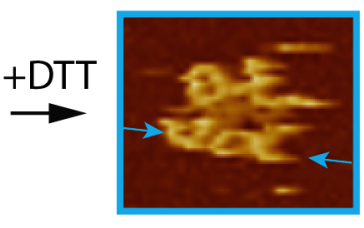

$4 \mathrm{~min}$

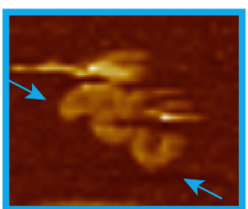

$8 \mathrm{~min}$

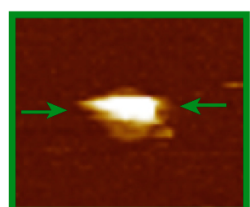

15 min

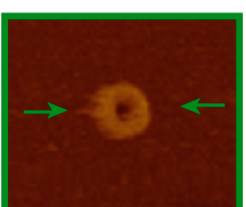

$20 \min$

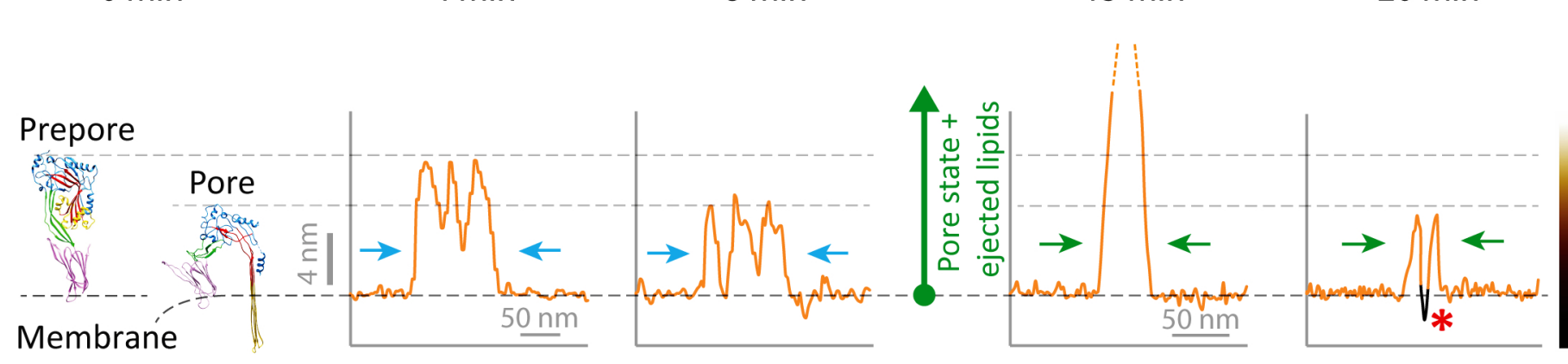

Figure 4
0 min

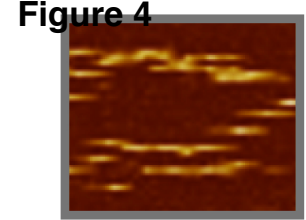

Membrane 
Video 1
Click he

Click here to download Video: Video1.mp4

Click here to download Video: Video1.mp

4


Click here to download Video: Video2.mp4 Click here to download Video: Video2 mp4

(1)

(1)

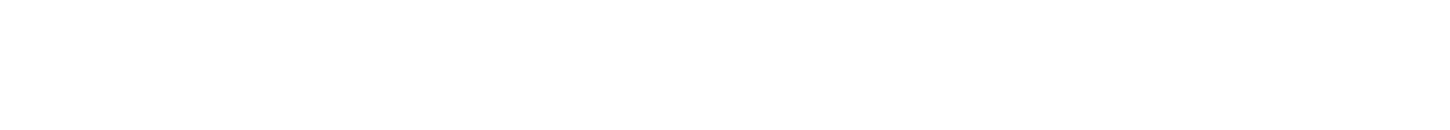

.

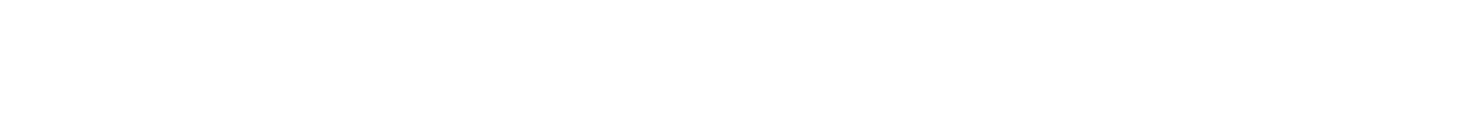

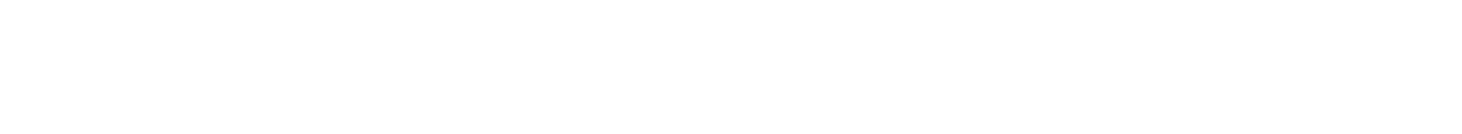

政

列

列

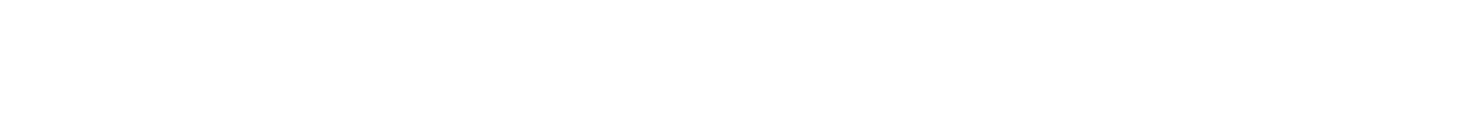

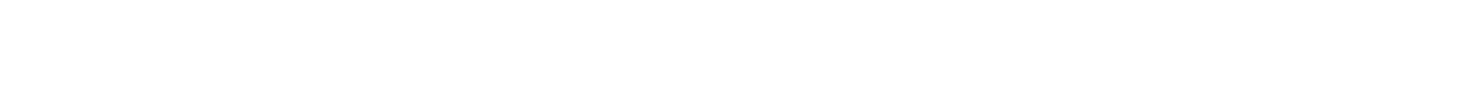

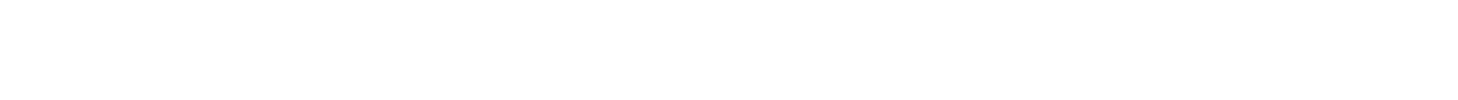

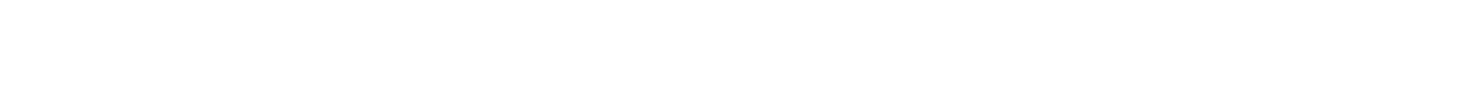

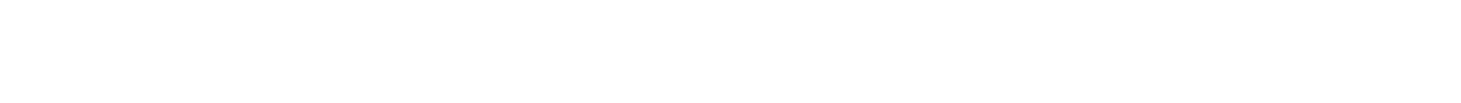

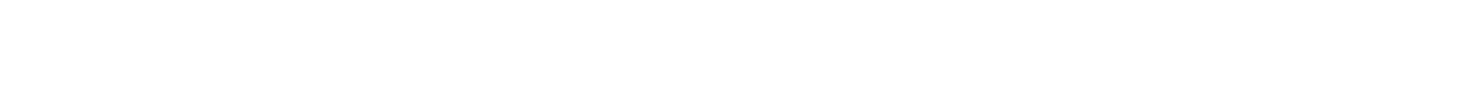

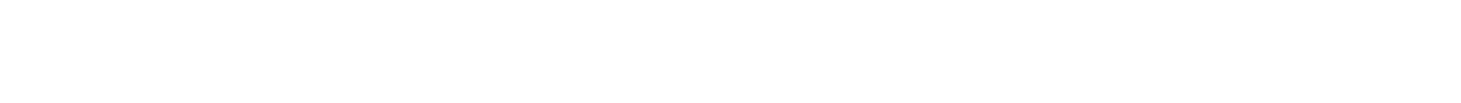


Click here to download high resolution image

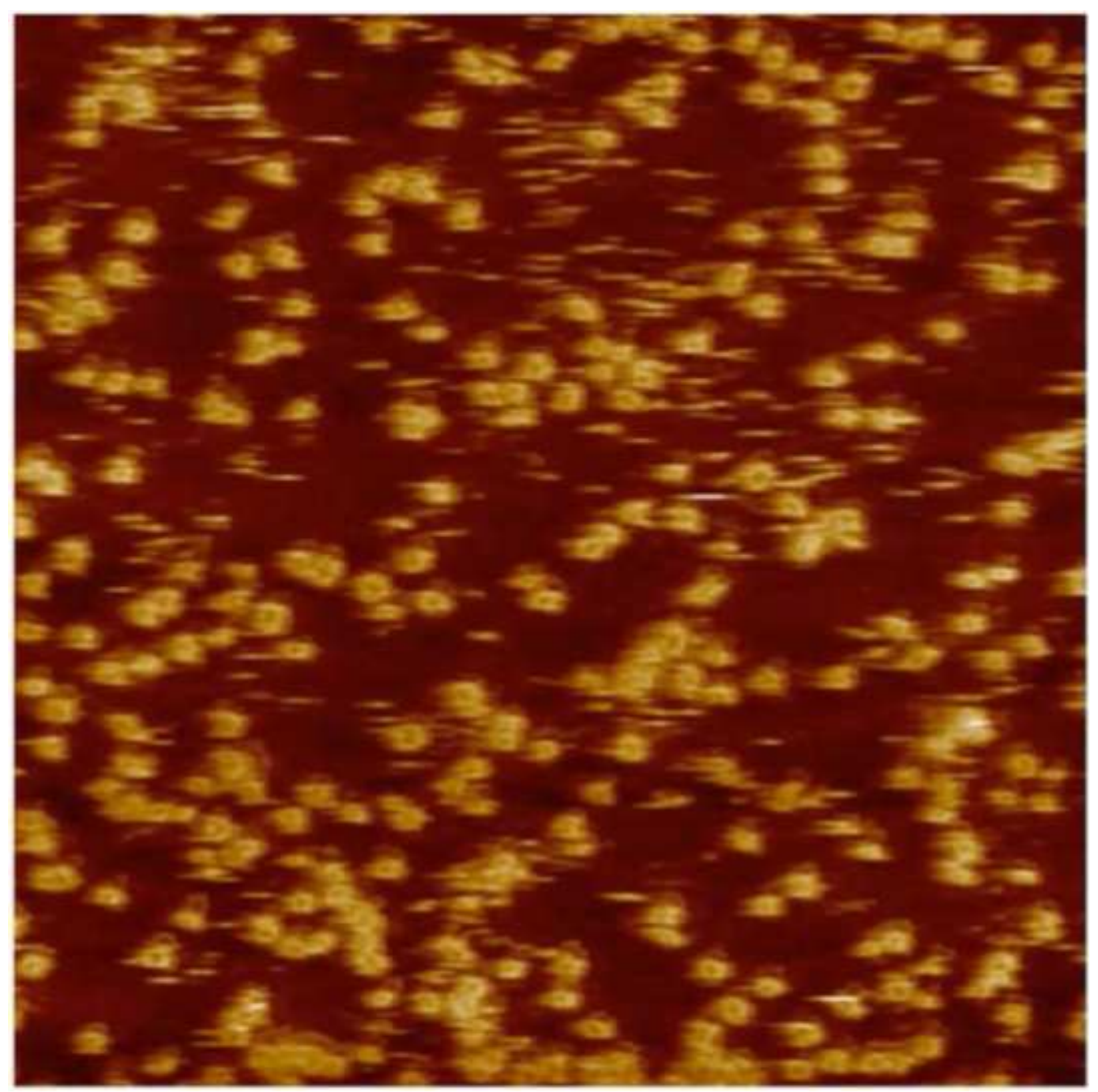

Video 1 Still

\section{click here to download high resolution image}


Video 2 Still
Click here to download high resolution image

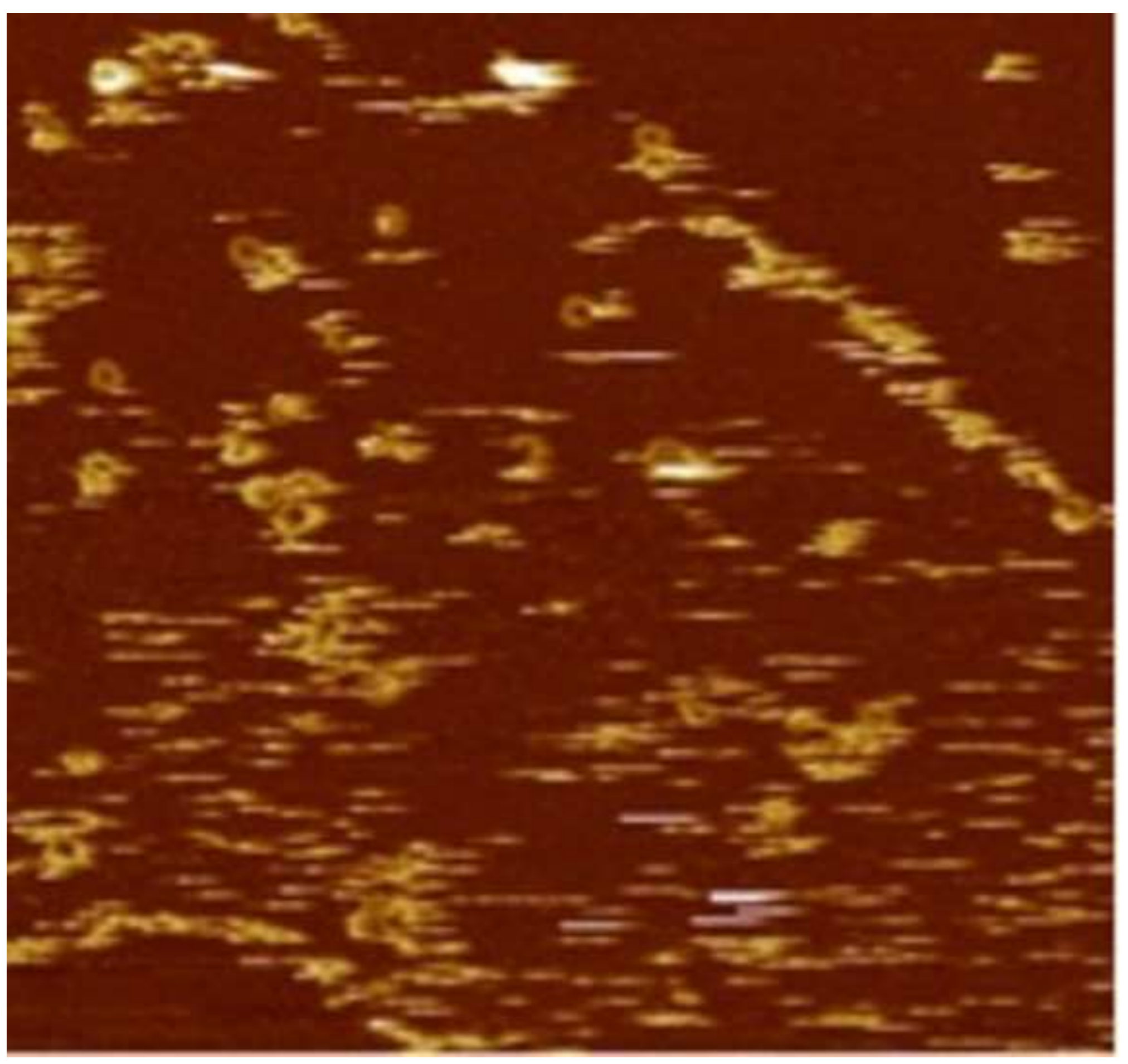

